\title{
TEACHING MEDICAL PARASITOLOGY IN A NEW PHYSICIAN ASSOCIATE MASTER'S PROGRAMME
}

\author{
A. Peña-Fernández ${ }^{1}$, M.A. Peña ${ }^{2}$ \\ ${ }^{1}$ De Montfort University, Faculty of Health and Life Sciences (UNITED KINGDOM) \\ ${ }^{2}$ Universidad de Alcalá, Departamento de Ciencias Biomédicas (SPAIN)
}

\begin{abstract}
The programme of Physician Associate (PA) is a relatively new Master's (MSc) programme in the United Kingdom (UK) to train future health care professionals that will work under the supervision of a medical doctor as part of a medical team. Although PAs cannot prescribe, they will provide crucial support to doctors by dealing with patients in general practitioner surgeries or hospitals. The UK Department of Health (DoH) in collaboration with the Royal College of Physicians and the Royal College of General Practitioners has established the Competence and Curriculum Framework for the physician associate qualification. The minimum length of the programme is 90 weeks, equivalent to 3,150 hours of nominal study time, with a minimum of 1,600 hours assigned to clinical learning. The $\mathrm{DoH}$ has also published the matrix specification of core clinical requirements for the PA, in which the competences for diagnosing and managing different medical conditions are described. In relation to human parasitic infections, the core clinical conditions for PAs have been divided into different categories as follows: $1 \mathrm{~A}$ ) is able to diagnose and manage, without referral, threadworms (Enterobius vermicularis) and hookworms (Ancylostoma duodenale and Necator americanus); 1B) is able to identify and take measures to avoid immediate deterioration of amebiasis (Entamoeba histolytica) and malaria (Plasmodium spp.); and 2B) is able to undertake the day-to-day management of the patient when diagnoses has been made for toxoplasmosis (Toxoplasma gondii).
\end{abstract}

De Montfort University (DMU, Leicester, UK) has launched the MSc Physician Associate Studies as part of its curricula in 2017/18. In order to develop clinical medical parasitology skills and reflection/critical thinking of the PA students, we have developed and implemented a two hour workshop in the module "Community Health", in which PA students $(n=12)$ have used the novel website resource DMU e-Parasitology to resolve two highly interactive virtual clinical case studies related to Entamoeba histolytica and Plasmodium spp. The DMU e-Parasitology, which is being developed by three European universities, is aimed at facilitating the teaching and learning of parasitology and it is publicly available through the DMU website: http://parasitology.dmu.ac.uk. Briefly, the virtual case studies provide the user with a medical history of a patient and different clinical slides to diagnose parasitic diseases by their identifiable structures and characteristics using a virtual microscope. The user will navigate through different interactive questions and mini quizzes to reach diagnoses, management, treatment and tailor prevention techniques for the case study proposed. The workshop was well-received by PA students, as evidenced by their favourable evaluation. The PA students that completed the voluntary questionnaire indicated that the design used to build the module of the virtual case studies was appropriate (50\% agreed, $50 \%$ strongly agreed). A similar percentage reported that the exercises and assessments presented were easy to understand and appropriate for their studies $(75 \%$ agreed, $25 \%$ strongly agreed). Finally, participants indicated that they gained appropriate knowledge of the pathology, prevention and treatment of malaria and amebiasis $(25 \%$ agreed, $75 \%$ strongly agreed). This paper describes tools, methods and strategies that can be easily adopted for implementing new ways of teaching and learning medical parasitology and clinical skills.

Keywords: Physician associate, virtual clinical case studies, medical parasitology.

\section{INTRODUCTION}

The specific programme to train mid-level practitioners' physician assistants in the United States has been introduced in the United Kingdom (UK) in form of Master (MSc) of Physician Associate (PA) relatively recently with the first batch of graduates in 2009 [1]. In a few years, the MSc PA has become a highly desirable programme for any graduated non-medical health care student that wishes to work in a medical team under the supervision of a medical doctor. In the UK, the PA programme is a twoyear full-time course whose competences and curriculum framework are defined by the Department of 
Health (2012, [2]) in collaboration with the Royal College of Physicians and the Royal College of General Practitioners.

The Department of Health (2006, [3]) also has published the matrix specification of core clinical requirements for the $\mathrm{PA}$, in which the competences for diagnosing and managing different medical conditions are described. The core clinical conditions that PAs are required to learn regarding human parasites include the following categories:

- $1 \mathrm{~A})$ in which the future PA is able to diagnose and manage, without referral, threadworms (Enterobius vermicularis) and hookworms (Ancylostoma duodenale and Necator americanus).

- 1B) the PA will be able to identify and take measures to avoid immediate deterioration of amebiasis (Entamoeba histolytica) and malaria (Plasmodium spp.).

- $2 \mathrm{~B}$ ) the future PA is able to undertake the day-to-day management of the patient when a diagnosis has been made for toxoplasmosis (Toxoplasma gondii).

\subsection{Physician associate programme at De Montfort University}

De Montfort University (DMU, Leicester, UK) launched the MSc Physician Associate Studies in 2017/18 and the first cohort of DMU students has recently undertaken their exams to pass into the second year in which they will need to complete a minimum of 1,600 hours assigned to clinical learning and complete an evidence-based, practice-related project (60 credits). A description of the structure and course modules is available at the DMU PA website ([4]; Figure 1).
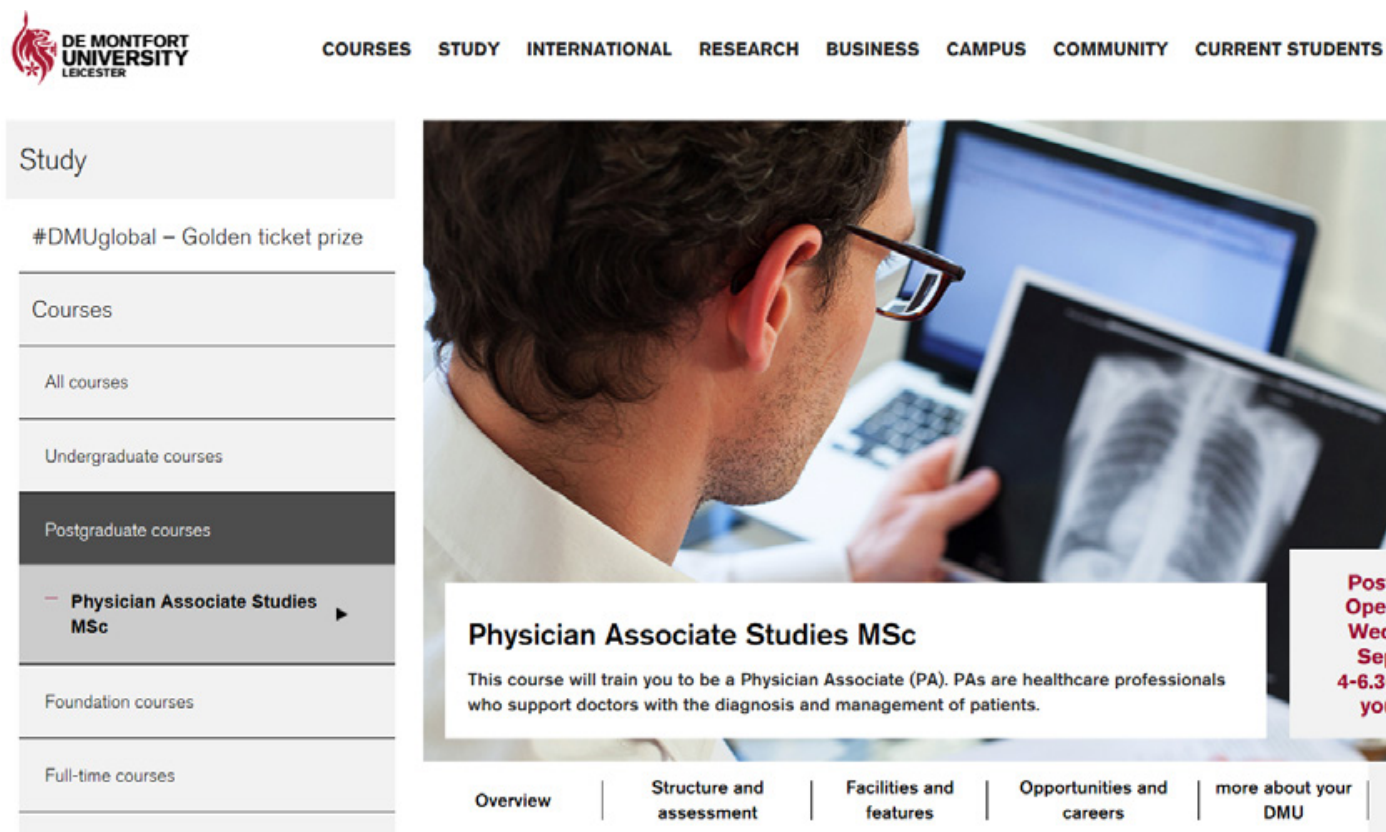

Figure 1. Overview of the De Montfort University Physician Associate Studies MSc website. Available at: http://www.dmu.ac.uk/study/courses/postgraduate-courses/physician-associate-studies-msc/physicianassociate-studies.aspx

A total of 12 students were enrolled in the programme in 2017/18, and they needed to complete the following five modules: Anatomy and Physiology, Community Health, Clinical \& Professional Skills, and General Medicine I and II. The content related to parasitic diseases was delivered in the 15 credit module of "Community Health". In order to facilitate the acquisition of some clinical medical parasitology skills and to promote reflection and critical thinking, PA students we provided with 5 hours of theoretical and practical content as follows:

a) Theoretical content $\rightarrow$ totalling 3 hours. The parasitology content was delivered using traditional methods in combination with clinical mini-case studies and mini-quizzes, as we have found previously that this pedagogic strategy would seem to be successful in facilitating the acquisition of some basic clinical skills when teaching parasitology topics to final year Biomedical Science undergraduate students [5]. Parasitology mini-case studies were created in 
collaboration with professional parasitologists and academics following our previous experience with BMS students but enhancing their difficulty. We also followed the scaffolding and structure of the monthly case studies described in the Laboratory Identification of Parasitic Diseases of Public Health Concern (DPDx) (CDC, 2017, [6]). Briefly, mini-case studies are provided with a short medical history and real clinical slides from our laboratories and/or CDC. PA students, working in pairs, provided a possible "diagnosis" for each mini-case study based on the medical history of the patient in conjunction with the morphological features of the specimens provided. PA students were encouraged to focus on the medical history and management of the patient and to reflect on the information provided during the lectures to suggest potential diagnoses, further tests and management of the patient presented.

In addition to the mini-case studies, students were also requested to complete a few quizzes regarding the topics delivered within the lectures as described in Figure 2.

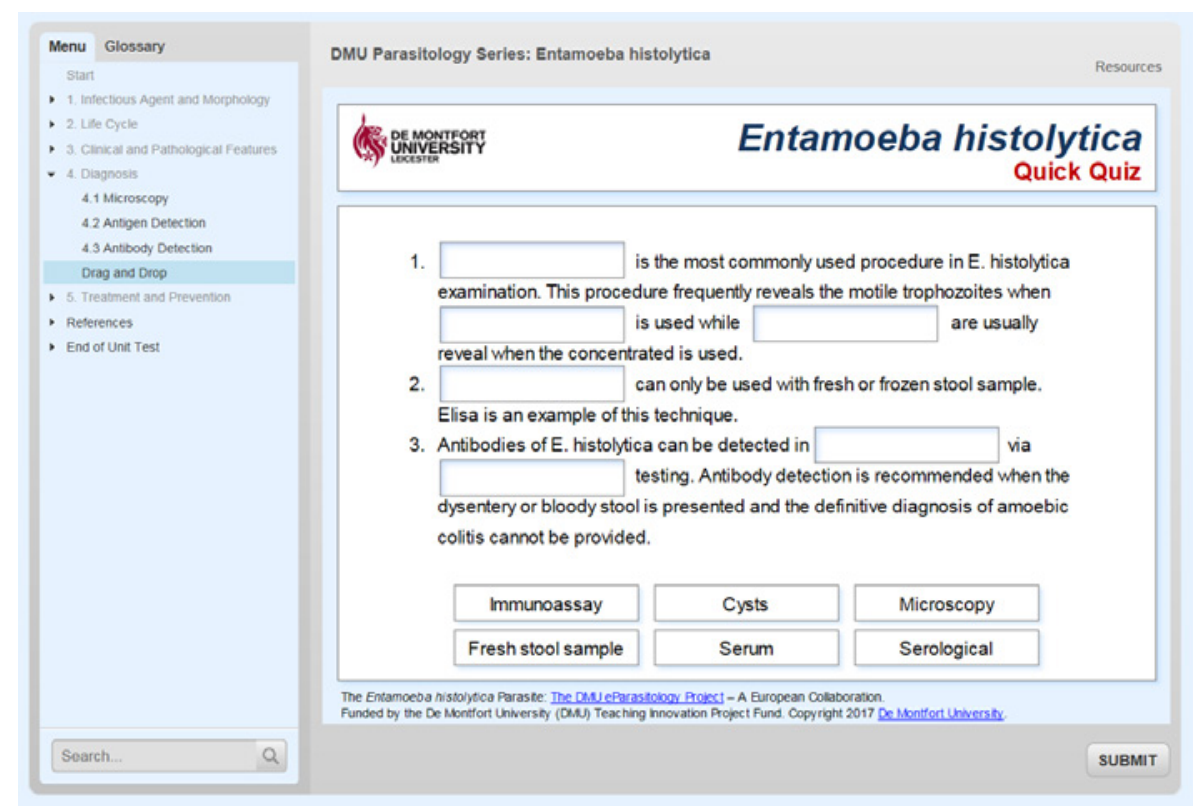

Figure 2. Overview of one of the mini-quizzes presented. Image taken from the Entamoeba histolytica unit in the DMU e-Parasitology (Image courtesy of DMU). Available at: http://parasitology.dmu.ac.uk/learn/modules/entamoeba/story_htm/5.html

b) Workshop $\rightarrow$ PA students were provided with a two-hour workshop in which they needed to complete two highly interactive virtual clinical case studies developed for the novel DMU eParasitology package. The DMU e-Parasitology package is an international project led by De Montfort University; and a complete description of the structure and methods used to develop the virtual clinical case studies has been recently described in Peña-Fernández et al. (2018) [7].

We selected the virtual clinical case studies related to Entamoeba histolytica and malaria, as the PA needs to be able to identify these pathogens, so they could benefit from using the DMU e-Parasitology resources of virtual library and microscope to learn to identify these parasites.

\section{AIMS}

The objectives of this communication are to initially test the DMU e-Parasitology Virtual Case Studies module with PA students as well as to investigate the relative success of the different teaching approaches used in conjunction with this innovative package to promote acquisition of basic parasitology skills and knowledge. This study will also provide us with feedback to improve this DMU e-Parasitology module, which is critical for promoting self-learning and acquisition of clinical parasitology skills. 


\section{METHODS}

Briefly, students working in pairs completed two virtual clinical case studies with different levels of difficulty in a computer lab room, one per hour, as we left 10 minutes discussion at the end of each case to discuss the results al together to cement knowledge. Students were provided with a short introduction of the DMU e-Parasitology so they were able to become familiar with it. They started the case study with a recorded level of difficulty of 'easy'; and the package is user-friendly so students are able to quickly navigate through the different sections and modules. To promote engagement, case studies display different questions, mini-quizzes and formative assessments in an engaging and highly interactive manner. Thus, for example, different clinical slides are provided in which the user will be required to use the virtual microscope for identifying the parasites. The user will navigate through different interactive questions and mini quizzes to reach diagnoses, management, treatment and tailor prevention techniques for the case study proposed.

At the end of the workshop session, students were provided with a validated feedback-questionnaire with Likert scale and open-questions (free-response) to collect their impressions and opinion on the structure, use and format of the DMU e-Parasitology package but specifically for the Virtual Clinical Case Studies module developed to date. PA students' feedback and suggestions will be used to enhance this module to be launch in 2019. Ethical approval was provided by the Research Ethics Committee at DMU (Ref. 1851; 8th December 2016).

\section{RESULTS}

Only four students out of the 12 appropriately completed the questionnaire and were used for this analysis. The workshop was well-received by PA students, as evidenced by their favourable evaluation. Thus, all participants indicated that they enjoyed and were, from a general viewpoint, satisfied with the DMU e-Parasitology (Figure 3).
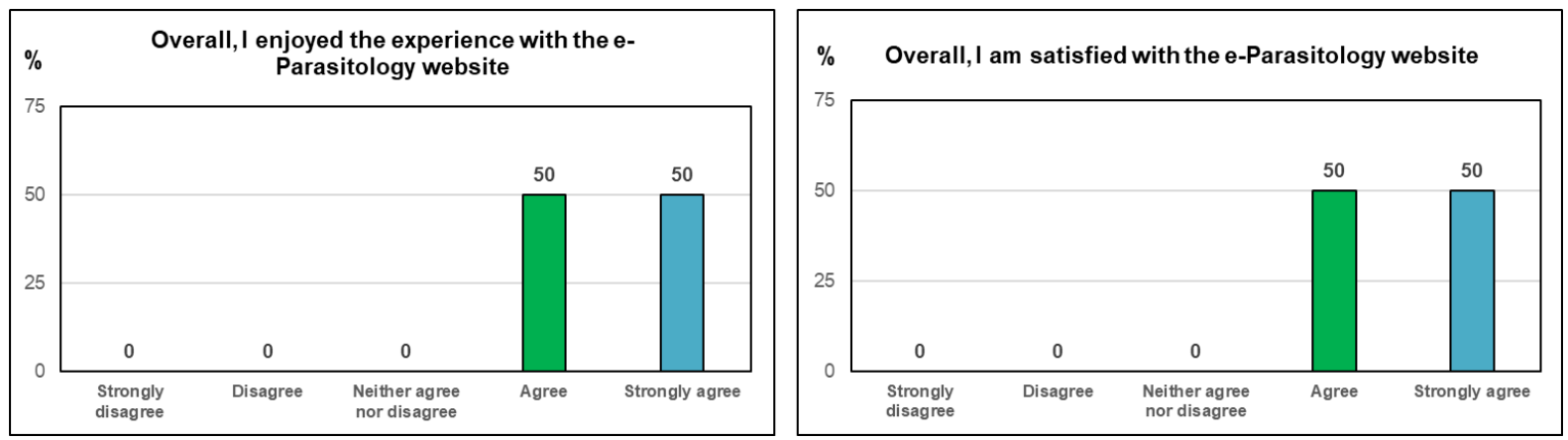

Figure 3. Students' enjoyment and satisfaction of the DMU e-Parasitology (\%).

Regarding the design and scaffolding of the DMU e-Parasitology Virtual Case Studies module, participants indicated that it was appropriate (50\% agreed, $50 \%$ strongly agreed); and similar percentages were recorded in relation to the navigation between the different modules and units. Thus, all students indicated that the overall design of the package was appropriate and engaging (Figure 4).
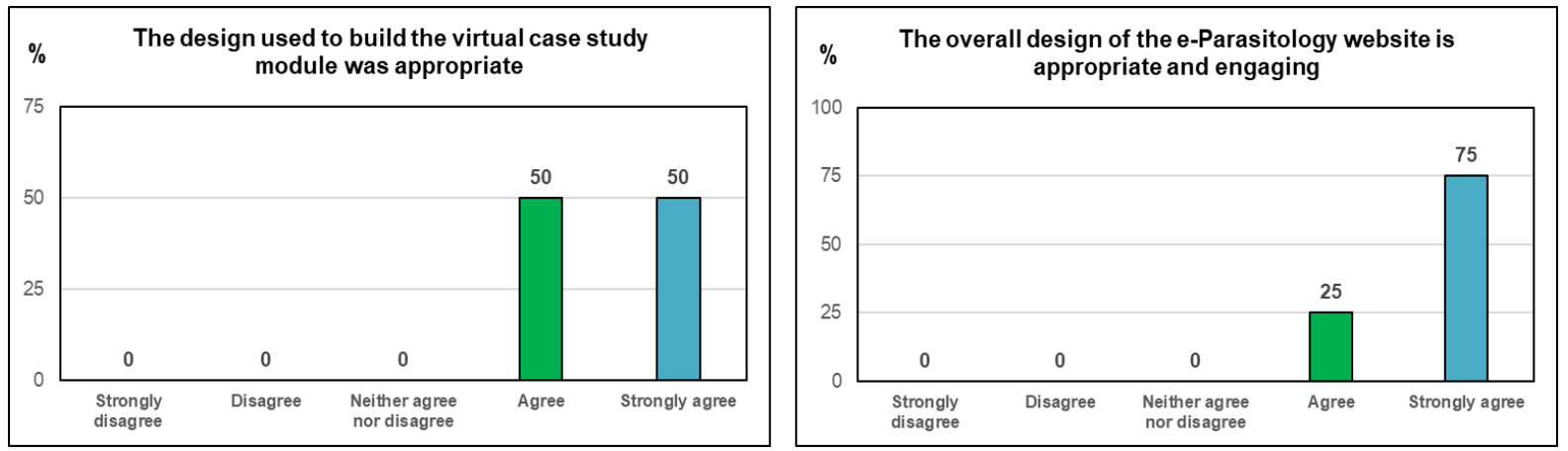

Figure 4. Students' opinions on the design of the DMU e-Parasitology (\%). 
A similar percentage of participants reported that the exercises and assessments presented were easy to understand (Fig. 5) and appropriate for their studies ( $75 \%$ agreed, $25 \%$ strongly agreed), which in turn enhanced their learning (Fig. 5).
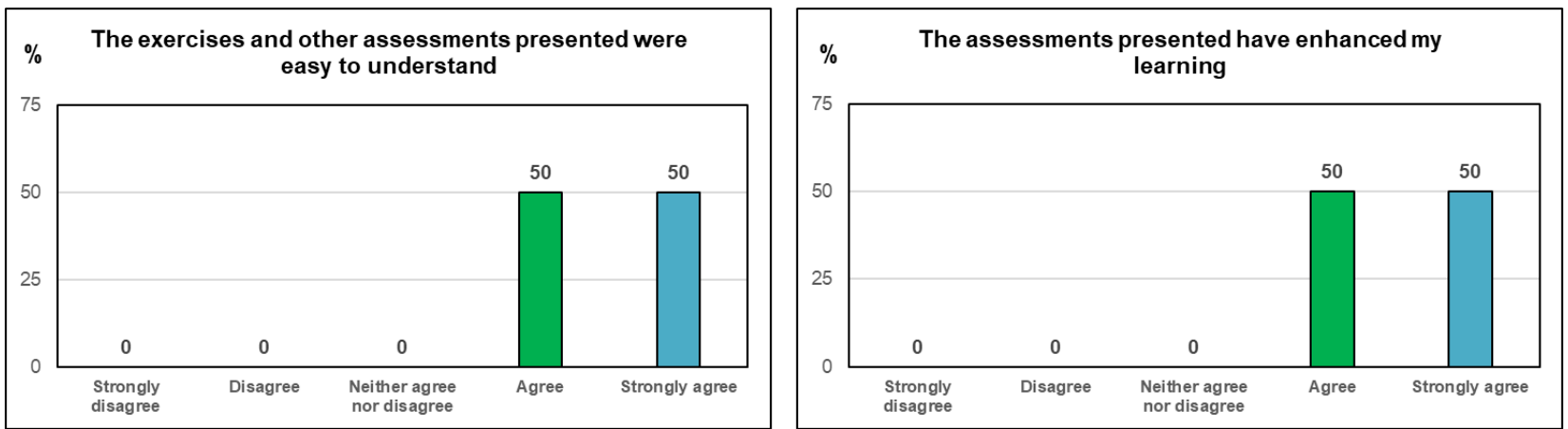

Figure 5. Students' opinions on the difficulty of the quizzes and assessments presented in the case studies and their impact on learning (\%).

Students indicated that by completing the two virtual clinical case studies they gained appropriate knowledge of the pathology, prevention and treatment of amebiasis and malaria (Fig. 6). Unfortunately, and owing to the fact that we did not record the parasitology knowledge before and after of our short pedagogic intervention, we are unable to define whether our intervention had a positive impact in the acquisition of some parasitological skills and/or knowledge. Our innovative teaching group will investigate this in future studies.

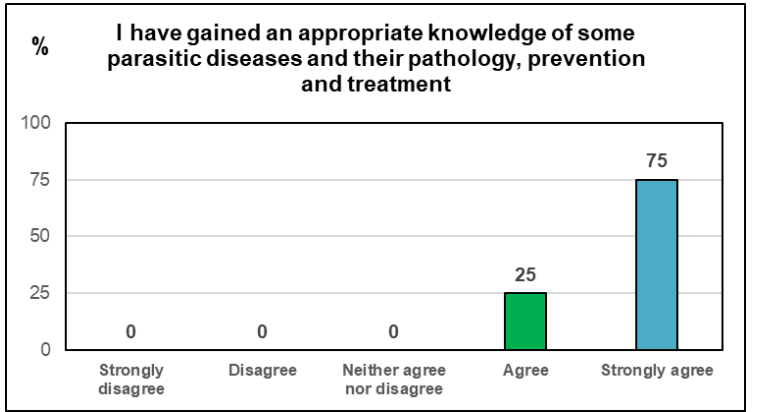

Figure 6. Students' opinions on acquisition of knowledge after completing the two virtual case studies (\%).

Finally, and as displayed in Figure 7, participants have indicated that the package and the workshop performed have enhanced their interest in the study of parasitology; and some of them would have also suggested an increment in the number of hours dedicated to the study of human parasites in the PA programme.
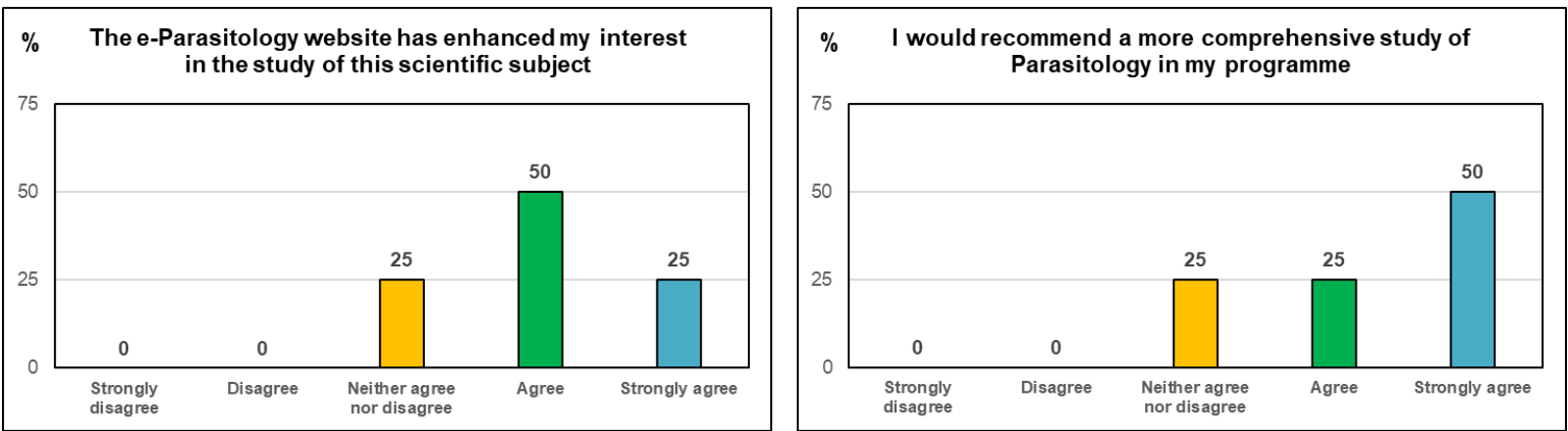

Figure 7. Students' opinions on their interest in studying parasitology after using the DMU e-Parasitology package and about the status of parasitology in their programme (\%). 


\section{CONCLUSIONS}

Although our results should be taken carefully due to the very small number of participants that have provided feedback, in general, PA students enjoyed and were satisfied with the workshop and the innovative clinical case studies performed. These results are highlighted by the fact that these students have recommended an increase in the teaching of parasitology in their programme, which is a significant comment as the PA is an intensive course with a myriad of different topics that students need to acquire in a very short period of time. More studies are needed to explore if the methods used have an impact in the acquisition of knowledge and parasitology skills. This paper describes tools, methods and strategies that can be easily adopted for enhancing the teaching of medical parasitology to postgraduate students with different backgrounds on parasitology.

\section{ACKNOWLEDGEMENTS}

The authors would like to express their sincere appreciation to Jonathan Coope and Maxine Armstrong (DMU) for work on development of DMU e-Parasitology and to USP-CEU Parasitology laboratory for providing high-quality images of parasites in different clinical and culture samples. Finally, we would like to thank the Teaching Innovation Project Fund at De Montfort University (scheme 2015-16) to fund this project to Dr. Peña-Fernández.

\section{REFERENCES}

[1] de Lusignan S., McGovern AP., Tahir MA., Hassan S., Jones S., Halter M., Joly L., Drennan VM. Physician Associate and General Practitioner Consultations: A Comparative Observational Video Study. PLoS One 2016; 11(8):e0160902.

[2] Department of Health. The Competence and Curriculum Framework for the Physician Assistant 2012. Available at: http://www.fparcp.co.uk/about-fpa/Who-are-physician-associates

[3] Department of Health. Matrix specification of Core Clinical Conditions for the Physician Assistant by category of level of competence, 2006. Available at: http://www.fparcp.co.uk/aboutfpa/Who-are-physician-associates

[4] De Montfort University. Physician Associate Studies MSc website, 2018. Available at: http://www.dmu.ac.uk/study/courses/postgraduate-courses/physician-associate-studies$\mathrm{msc} /$ physician-associate-studies.aspx

[5] Peña-Fernández A., loannou M., Lobo-Bedmar MC., Fenoy S. Curriculum modifications for teaching parasitology and infectious diseases in a Biomedical Science degree. ICERI2017 Proceedings; 1593-1598. ISBN: 978-84-697-6957-7.

[6] CDC (Centres for Disease Control and Prevention). Laboratory Identification of Parasitic Diseases of Public Health Concern (DPDx); Monthly Case Studies 2017. Available at: https://www.cdc.gov/dpdx/monthlyCaseStudies/2016/index.html [accessed 26/09/2018]

[7] Peña-Fernández A., Magnet A., Peña MA. Focus group to create a virtual case study model unit for the DMU e-Parasitology. EDULEARN18 Proceedings 2018; pp. 7104-7108. ISBN: 978-8409-02709-5. 